\title{
Pattern, Precipitants and Short Term Outcome of Heart Failure Patients Managed at Federal Medical Centre Nguru, a Tertiary Health Centre in Yobe State Northeastern Nigeria
}

\author{
Musa Mohammed Baba1 ${ }^{*}$, Faruk Buba ${ }^{2}$, Mohammed Abdullahi Talle², Habu Abdul ${ }^{1}$ \\ ${ }^{1}$ Department of Internal Medicine, Federal Medical Centre Nguru, Nguru, Nigeria \\ ${ }^{2}$ Department of Medicine, College of Medical Sciences, University of Maiduguri, Maiduguri, Nigeria \\ Email: ^drbaba01@gmail.com
}

How to cite this paper: Baba, M.M., Buba, F., Talle, M.A. and Abdul, H. (2018) Pattern, Precipitants and Short Term Outcome of Heart Failure Patients Managed at Federal Medical Centre Nguru, a Tertiary Health Centre in Yobe State Northeastern Nigeria. International Journal of Clinical Medicine, 9, 432-444.

https://doi.org/10.4236/ijcm.2018.95037

Received: March 19, 2018

Accepted: May 18, 2018

Published: May 21, 2018

Copyright $(9) 2018$ by authors and Scientific Research Publishing Inc. This work is licensed under the Creative Commons Attribution International License (CC BY 4.0).

http://creativecommons.org/licenses/by/4.0/

\begin{abstract}
Heart Failure (HF) is a syndrome characterized by typical symptoms (e.g. breathlessness, ankle swelling and fatigue) that may be accompanied by signs (e.g. elevated jugular venous pressure, pulmonary crackles and peripheral oedema) caused by a structural and/or functional cardiac abnormality, resulting in a reduced cardiac output and/or elevated intracardiac pressures at rest or during stress. Clinical studies investigating the aetiological patterns of heart failure in Sub-Saharan Africa (SSA) revealed that hypertension, cardiomyopathy and rheumatic heart disease account for more than two-thirds of cardiac disease cases. The objective of this study therefore is to assess the pattern, precipitating factors and short term outcome of heart failure among patients admitted into our hospital. Method: The study was a prospective cross-sectional type conducted among in patients with heart failure in the medical ward of the hospital. Results: A total of three and fifty four (354) subjects were recruited into the study, comprising one hundred and twenty nine males (36.4\%) and two hundred and twenty five (63.4\%) females. Majority of the patients were admitted in NYHA functional class IV. This however improved to class II at discharge though few were discharged in NYHA class III. Heart failure secondary to progressive hypertensive heart disease was the most common (39.0\%) followed by peripartum cardiomyopathy (22.6\%) while idiopathic dilated cardiomyopathy (11.0\%) and rheumatic heart disease (7.3\%) were the third and fourth causes of heart failure respectively. The most common precipitating factor for heart failure in this study was chest infection (44.9\%). In conclusion, the study revealed that progressive hypertensive heart disease was the leading cause of heart failure, followed by peripartum cardi-
\end{abstract}


omyopathy while idiopathic dilated cardiomyopathy and rheumatic heart disease were ranked third and fourth causes of heart failure respectively. Though myocardial infarction is on the increase, it was found to be the fifth cause of heart failure. The study also identified the following precipitants of heart failure in decreasing order of occurrence: chest infection, non-adherence to prescription, and urinary tract infection.

\section{Keywords}

Patterns, Precipitant, Short Term Outcome, Heart Failure

\section{Introduction}

Heart Failure (HF) is a clinical syndrome characterized by typical symptoms (e.g. breathlessness, ankle swelling and fatigue) that may be accompanied by signs (e.g. elevated jugular venous pressure, pulmonary crackles and peripheral oedema) caused by a structural and/or functional cardiac abnormality, resulting in a reduced cardiac output and/or elevated intracardiac pressures at rest or during stress [1]. The American College of Cardiology and American Heart Association (ACC/AHA) guidelines on heart failure classified the progression of heart disease into four stages as follows: Stage A patients are at high risk for heart failure but have no structural heart disease or symptoms of heart failure; Stage B patients have structural heart disease but have no symptoms of heart failure; Stage $\mathrm{C}$ patients have structural heart disease and have symptoms of heart failure and Stage D patients have refractory heart failure requiring specialized interventions [2]. Similarly, the New York Heart Association (NYHA) classified heart failure into four functional classes based on the relationship between dyspnoea and the amount of effort required to provoke this symptom, as follows: Class I patients have no limitation of physical activity; Class II patients have slight limitation of physical activity; Class III patients have marked limitation of physical activity and Class IV patients have symptoms even at rest and are unable to carry on any physical activity without discomfort [3]. Heart failure has also been classified according to the ejection fraction, natriuretic peptide levels and the presence of structural heart disease and diastolic dysfunction into three subtypes, namely HF with reduced ejection fraction (HFrEF) with left ventricular ejection fraction of $<40 \%$, HF with preserved ejection fraction (HFpEF) with left ventricular ejection fraction of $>50 \%$ and HF mid-range ejection fraction (HFmrEF) with left ventricular ejection fraction of $40 \%-49 \%$ [4]. It has emerged as the most common primary diagnosis for patients admitted to hospital with suspected cardiac disease in Africa [5].

A review of clinical studies investigating the aetiological patterns of heart failure in Sub-Saharan Africa (SSA) has revealed that hypertension, cardiomyopathy and rheumatic heart disease account for more than two-thirds of cardiac disease cases [5] [6]. A multicentred study for heart failure in Africa, "the 
sub-saharan Africa Survey of Heart Failure" (THESUS-HF) has shown a shift in the cardiovascular disease profile in the region [7]. The study characterised the causes, treatment and short-term outcome in 1006 Africans from nine sub-saharan African countries. It highlighted an increasing major contribution of hypertension as a cause of heart failure (from $23 \%$ to $43 \%$ ), an increasing importance of cardiomyopathies (from $20 \%$ to $29 \%$ ), a reduced recognition of rheumatic heart disease (from $22 \%$ to $17 \%$ ), and a rise in ischaemic heart disease (from $2 \%$ to $8 \%$ ) in the aetiology of heart failure. In a heart failure cohort study comprising sixteen countries, mortality was the highest in Africa and India, intermediate in Southeast Asia, and lowest in China, South America, and the Middle East [8]. Heart failure mortality was found to be greater than $50 \%$ for patients with New York Heart Association (NYHA) class IV or American College of Cardiology/American Heart Association (ACC/AHA) stage D [9] [10] [11]. Heart failure related to systolic dysfunction has an associated mortality of $50 \%$ after 5 years [12]. The objective of this study therefore is to assess the pattern, precipitating factors and short term outcome of heart failure among patients admitted into our hospital.

\section{Methodology}

The study was a prospective cross-sectional one conducted over a period of twelve months (from June 2016 to June 2017) among inpatients with heart failure in the medical ward of the hospital after obtaining ethical approval from the ethics and research committee of the hospital as well as verbal consent from the patients or their relatives. The inclusion criteria for the study is adult with heart failure symptoms that meet the Framingham diagnostic criteria while the exclusion criteria are patients with heart failure symptoms that does not meet the Framingham criteria [13] or patient with heart failure symptoms less than 18 years. All patients had detailed medical history and examination. Serum electrolytes, Fasting blood glucose, lipid profile, electrocardiography, and echocardiography were done to all patients. All patients were placed on same regimen of heart failure except otherwise contraindicated (Angiotensin converting enzyme inhibitor or Angiotensin receptor blocker, Aldosterone antagonist, Carvedilol and either Furosemide or Torsemide. Digoxin was added to patients with arrhythmias or those with poor systolic function. Amiodarone was however prescribed to patients with refractory atrial fibrillation. Anti-platelets and Warfarin were prescribed to patients with dilated cardiac chamber with low ejection fractions plus hypokinesia and those with rheumatic heart disease in atrial fibrillation respectively as part of heart failure management protocol [1].

\section{Data Analysis}

Statistical analysis was done using SPSS version 21.0 (SPSS IBM). Data was presented as mean \pm standard deviation (SD) for continuous variables, while categorical variables were expressed as frequencies and proportion. Student t-test 
was used to compare mean values of continuous variables, while Fisher's exact test was used in comparing categorical variables. A p value of $<0.05$ was considered significant.

\section{Results}

Three and fifty three subjects fulfilled the inclusion criteria and were recruited into the study, comprising one hundred and twenty eight males (36.2\%) and two hundred and twenty five $(63.7 \%)$ females. The mean age of the study population was $46.98 \pm 19.33$ years. However, male subjects were significantly older than their female counterpart $57.31 \pm 15.51$ years and $41.05 \pm 18.82$ years respectively $\mathrm{P}<0.001$. The mean duration of hospital stay was $7.95 \pm 5.05$ days. Table 1 shows the demographic and clinical characteristics of the subjects. Majority of the patients were admitted in NYHA functional class IV which however improved to class II at discharge though few were discharged in NYHA class III (Table 2). Heart failure secondary to progressive hypertensive heart disease is the most common cause of heart failure (39.0\%) followed by peripartum

Table 1. Demographic and clinical characteristics of the study population.

\begin{tabular}{cccc}
\hline Parameters & Males (128) & Females (225) & P-value \\
\hline Age & $57.31 \pm 15.51$ & $41.05 \pm 18.82$ & $<0.001^{*}$ \\
SBP & $133.62 \pm 24.15$ & $124.70 \pm 24.89$ & $0.001^{*}$ \\
DBP & $81.59 \pm 13.51$ & $77.51 \pm 14.57$ & $0.010^{*}$ \\
HR & $102.85 \pm 20.21$ & $105.25 \pm 16.77$ & 0.235 \\
DHS & $8.09 \pm 4.37$ & $7.90 \pm 5.41$ & 0.807 \\
Wt & $72.71 \pm 11.77$ & $66.82 \pm 15.97$ & $<0.001^{*}$ \\
Ht & $1.64 \pm 0.07$ & $1.60 \pm 0.09$ & $<0.001^{*}$ \\
BMI & $26.96 \pm 4.19$ & $25.88 \pm 4.96$ & $0.033^{*}$ \\
Na & $134.47 \pm 5.89$ & $137.32 \pm 5.55$ & 0.815 \\
K & $4.24 \pm 0.66$ & $4.37 \pm 3.61$ & 0.681 \\
Urea & $8.24 \pm 5.59$ & $7.69 \pm 3.07$ & 0.232 \\
Cr & $163.59 \pm 99.96$ & $134.09 \pm 87.04$ & $0.004^{*}$ \\
\hline
\end{tabular}

SBP = Systolic Blood Pressure, DBP = Diastolic Blood Pressure, HR = Heart Rate, DHS = Duration of Hospital Stay, $\mathrm{Wt}=\mathrm{Weight}, \mathrm{Ht}=$ Height, $\mathrm{BMI}=$ Body Mass Index, $\mathrm{Na}=$ Sodium, $\mathrm{K}=$ Potassium, $\mathrm{Cr}=\mathrm{Creati}-$ nine. ${ }^{*}$ Significant $\mathrm{P}$ Values.

Table 2. New York Heart Association Functional class of the study subjects on admission and on discharge.

\begin{tabular}{|c|c|c|c|}
\hline Parameters & NYHA on Admission & NYHA on Discharge & P-Value \\
\hline NYHA I & $0(0.0 \%)$ & $0(0.0 \%)$ & \\
\hline NYHA II & $0(0.0 \%)$ & $224(63.3 \%)$ & \\
\hline NYHA III & $23(7.9 \%)$ & $111(31.4 \%)$ & \\
\hline NYHA IV & $326(91.8 \%)$ & $19(5.4 \%)$ & Fishers exact $\mathrm{P}$ value $<0.001^{\star \star *}$ \\
\hline
\end{tabular}

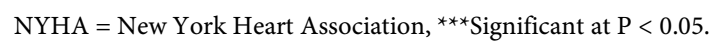


cardiomyopathy (22.6\%) while idiopathic dilated cardiomyopathy (11.0\%) and rheumatic heart disease $(7.3 \%)$ were the third and fourth causes of heart failure respectively (Table 3 ).

The most common precipitating factor for heart failure in this study was chest infection (44.9\%), followed by non-adherence to prescription (21.8\%). Urinary tract infection was the third ranked precipitant of heart failure (11.3\%). Arrhythmia especially atrial fibrillation was common among the patient with rheumatic heart disease. Table 4 shows the precipitating factors for heart failure among the study population. Impaired relaxation was the most common form diastolic dysfunction among heart failure secondary to progressive hypertensive heart disease while restrictive pattern of diastolic dysfunction was the dominant form among patients with peripartum cardiomyopathy and idiopathic dilated cardiomyopathy. Table 5(a) and Table 5(b) show the patterns of left ventricular diastolic function among the study population in relation to the respective diagnosis on admission and at discharge respectively. Majority of patients with hypertensive heart disease in failure had preserved left ventricular systolic function while patients with peripartum cardiomyopathy and idiopathic dilated cardiomyopathy had reduced left ventricular systolic function.

Table 3. Causes of Heart failure among the study subjects.

\begin{tabular}{cc}
\hline Diagnosis & Number of Cases (\%) \\
Hypertensive Heart Disease in failure & $138(39.0 \%)$ \\
Hypertensive/Diabetic in heart failure & $62(17.5 \%)$ \\
Peripartum cardiomyopathy & $80(22.6 \%)$ \\
Idiopathic dilated cardiomyopathy & $39(11.0 \%)$ \\
Rheumatic heart disease & $26(7.6 \%)$ \\
Myocardial infarction & $5(1.4 \%)$ \\
Cor-pulmonale & $1(0.3 \%)$ \\
\hline
\end{tabular}

Table 4. Precipitating factors for heart failure.

\begin{tabular}{cc}
\hline Precipitating Factor & Number of cases (\%) \\
Chest infection & $159(44.9 \%)$ \\
Non adherence to prescription & $77(21.8 \%)$ \\
Urinary tract infection & $40(11.3 \%)$ \\
Arinary tract infection with chest infection & $17(4.8 \%)$ \\
Chest infection with arrhythmia & $13(3.7 \%)$ \\
Urinary tract infection with arrhythmia & $27(7.6 \%)$ \\
Anaemia with uncontrolled hypertension & $8(2.3 \%)$ \\
Ischaemic heart disease & $8(2.3 \%)$ \\
Endocarditis & $3(0.8 \%)$ \\
\end{tabular}


Table 5. (a) Patterns of left ventricular diastolic function in relation to the respective diagnosis on admission; (b) Patterns of left ventricular diastolic function in relation to the respective diagnosis at discharge.

(a)

\begin{tabular}{ccccc}
\hline Diagnosis & Normal & Impaired relaxation & Pseudonormalisation & Restrictive pattern \\
\hline HHD & $32(23.2 \%)$ & $90(65.2 \%)$ & $0(0.0 \%)$ & $10(7.2 \%)$ \\
HT/DM & $5(8.1 \%)$ & $57(91.9 \%)$ & $0(0.0 \%)$ & $0(0.0 \%)$ \\
PPCM & $3(3.8 \%)$ & $0(0.0 \%)$ & $0(0.0 \%)$ & $72(90.0 \%)$ \\
IDCM & $2(5.1 \%)$ & $0(0.0 \%)$ & $0(0.0 \%)$ & $35(89.7 \%)$ \\
RHD & $5(19.2 \%)$ & $0(0.0 \%)$ & $0(0.0 \%)$ & $17(65.4 \%)$ \\
MI & $0(0.0 \%)$ & $5(100.0 \%)$ & $0(0.0 \%)$ & $0(0.0 \%)$
\end{tabular}

HHD = Hypertensive Heart Disease, HT/DM = Hypertensive/Diabetic, PPCM = Peripartum Cardiomyopathy, IDCM = Idiopathic Dilated Cardiomyopathy, RHD = Rheumatic Heart Disease, $\mathrm{MI}=$ Myocardial Infarction.

(b)

\begin{tabular}{ccccc}
\hline Diagnosis & Normal & Impaired relaxation & Pseudonormalisation & Restrictive pattern \\
\hline HHD & $33(23.9 \%)$ & $89(64.5 \%)$ & $0(0.0 \%)$ & $10(7.2 \%)$ \\
HT/DM & $5(8.1 \%)$ & $57(91.9 \%)$ & $0(0.0 \%)$ & $0(0.0 \%)$ \\
PPCM & $3(3.8 \%)$ & $0(0.0 \%)$ & $0(0.0 \%)$ & $72(90.0 \%)$ \\
IDCM & $2(5.1 \%)$ & $0(0.0 \%)$ & $0(0.0 \%)$ & $35(89.7 \%)$ \\
RHD & $5(19.2 \%)$ & $0(0.0 \%)$ & $0(0.0 \%)$ & $17(65.4 \%)$ \\
MI & $0(0.0 \%)$ & $5(100.0 \%)$ & $0(0.0 \%)$ & $0(0.0 \%)$ \\
& Cor-pulmonale (Only one patient and had no echocardiography report)
\end{tabular}

HHD = Hypertensive Heart Disease, HT/DM = Hypertensive/Diabetic, PPCM = Peripartum Cardiomyopathy, IDCM = Idiopathic Dilated Cardiomyopathy, RHD = Rheumatic Heart Disease, MI $=$ Myocardial Infarction.

Table 6(a) and Table 6(b) show the categories of left ventricular systolic function among the study population in relation to the respective diagnosis on admission and at discharge respectively. There was significant difference in the left ventricular systolic function on admission and at discharge among the study population particularly those admitted in heart failure with reduced ejection fraction irrespective of the cause of heart failure. However, no significant difference was observed in the left ventricular diastolic function on admission and at discharge. Table 7 shows the left ventricular functions on admission and at discharge among the study population irrespective of the cause of heart failure. Figure 1 shows the Doppler echocardiographic image of hypertensive disease with impaired relaxation pattern of diastolic dysfunction. Figure 2 shows Doppler echocardiographic image of restrictive pattern of left ventricular diastolic dysfunction in peripartum cardiomyopathy and Idiopathic dilated cardiomyopathy while Figure 3 shows two-dimensional echocardiographic image in rheumatic heart disease with thickened mitral valves and dilated left atrium. 
Table 6. (a) Categories of left ventricular systolic function in relation to the respective diagnosis on admission; (b) Categories of left ventricular systolic function in relation to the respective diagnosis at discharge.

(a)

\begin{tabular}{cccc}
\hline Diagnosis & HFpEF & HFrEF & HFmrEF \\
\hline HHD & $103(74.6 \%)$ & $25(18.8 \%)$ & $9(6.5 \%)$ \\
HT/DM & $62(100.0 \%)$ & $0(0.0 \%)$ & $0(0.0 \%)$ \\
PPCM & $2(2.5 \%)$ & $68(85.0 \%)$ & $10(12.5 \%)$ \\
IDCM & $0(0.0 \%)$ & $39(100.0 \%)$ & $0(0.0 \%)$ \\
RHD & $24(92.5 \%)$ & $0(0.0 \%)$ & $2(7.7 \%)$ \\
MI & $0(0.0 \%)$ & $5(100.0 \%)$ & $0(0.0 \%)$ \\
\multicolumn{2}{c}{ Cor-pulmonale: (Only one patient had and no echocardiography report) } \\
\hline
\end{tabular}

HHD = Hypertensive Heart Disease, HT/DM = Hypertensive/Diabetic, PPCM = Peripartum Cardiomyopathy, IDCM = Idiopathic Dilated Cardiomyopathy, RHD = Rheumatic Heart Disease, $\mathrm{MI}=$ Myocardial Infarction, HFpEF $=$ Heart Failure with preserved Ejection Fraction, HFrEF $=$ Heart Failure with reduced Ejection Fraction, HFmrEF = Heart Failure with mid-range Ejection Fraction.

(b)

\begin{tabular}{cccc}
\hline Diagnosis & HFpEF & HFrEF & HFmrEF \\
\hline HHD & $103(74.6 \%)$ & $25(18.8 \%)$ & $9(6.5 \%)$ \\
HT/DM & $62(100.0 \%)$ & $0(0.0 \%)$ & $0(0.0 \%)$ \\
PPCM & $11(13.8 \%)$ & $41(51.3 \%)$ & $28(35.0 \%)$ \\
IDCM & $0(0.0 \%)$ & $37(94.9 \%)$ & $2(5.1 \%)$ \\
RHD & $25(96.2 \%)$ & $0(0.0 \%)$ & $1(3.8 \%)$ \\
MI & $0(0.0 \%)$ & $4(100.0 \%)$ & $1(20.0 \%)$ \\
\multicolumn{2}{c}{ Cor-pulmonale: (Only one patient had and no echocardiography report) } \\
\hline
\end{tabular}

HHD = Hypertensive Heart Disease, HT/DM = Hypertensive/Diabetic, PPCM = Peripartum Cardiomyopathy, IDCM = Idiopathic Dilated Cardiomyopathy, RHD $=$ Rheumatic Heart Disease, $\mathrm{MI}=$ Myocardial Infarction, HFpEF = Heart Failure with preserved Ejection Fraction, HFrEF $=$ Heart Failure with reduced Ejection Fraction, $\mathrm{HFmrEF}=$ Heart Failure with mid-range Ejection Fraction.

Table 7. Left ventricular functions on admission and at discharge.

\begin{tabular}{cccc}
\hline Left ventricular functions & On Admission & At Discharge & P-value \\
\hline LV Systolic Function & & & \\
HFpEF $($ EF $>50 \%)$ & $192(54.4 \%)$ & $202(57.2 \%)$ & \\
HFmrEF (EF 40\% - 49\%) & $21(5.9 \%)$ & $41(11.6 \%)$ & Chi square $=0.006^{* *}$ \\
HFrEF (EF $<40 \%)$ & $137(38.8 \%)$ & $107(30.3 \%)$ & \\
LV Diastolic Function & & & \\
Normal LV Diastolic Function & $47(13.3 \%)$ & $48(13.6 \%)$ & \\
Impaired Relaxation pattern & $152(43.1 \%)$ & $151(42.8 \%)$ & Chi square $=0.993$ \\
Pseudonormalised pattern & $0(0.0 \%)$ & $0(0.0 \%)$ & \\
Restrictive pattern & $134(38.0 \%)$ & $134(38.0 \%)$ & \\
\hline
\end{tabular}

HFpEF $=$ Heart Failure with preserved Ejection Fraction, HFmrEF = Heart Failure with mid-range Ejection Fraction, HFrEF $=$ Heart Failure with reduced Ejection Fraction and ${ }^{* *}=$ Significant at $\mathrm{P}<0.05$. 


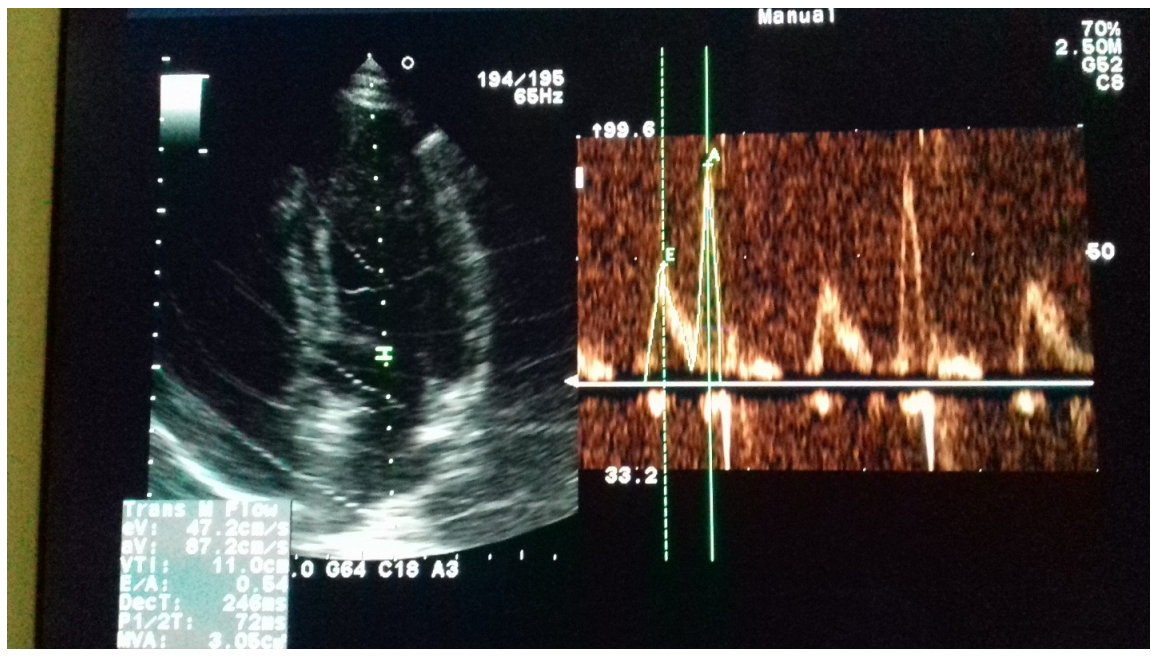

Figure 1. Doppler Echocardiography showing impaired relaxation pattern of diastolic dysfunction with reduced $\mathrm{E} / \mathrm{A}$ ratio in patient with progressive hypertensive heart disease.

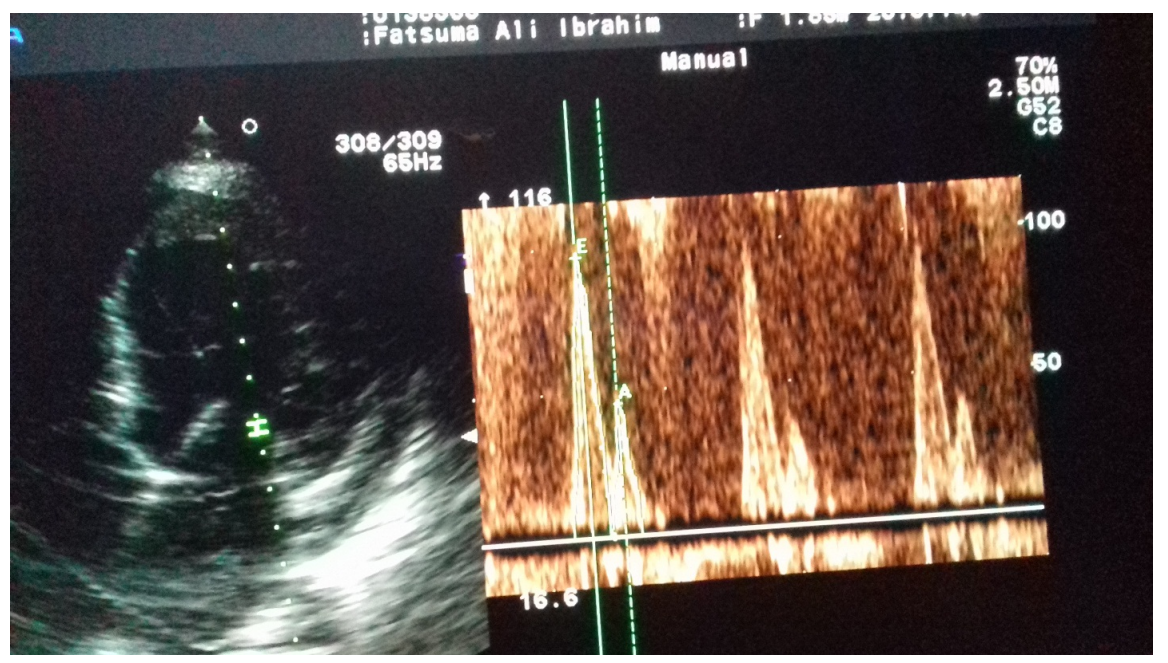

Figure 2. Doppler Echocardiography showing restrictive pattern of diastolic dysfunction with increased E/A ratio seen in peripartum cardiomyopathy and idiopathic dilated cardiomyopathy.

Of the three hundred and fifty three patients admitted in the ward, 319 (90.1\%) were discharged with improved clinical condition, 19 (5.4\%) died on admission, $11(3.1 \%)$ were discharged on request due to financial constraints while $3(0.8 \%)$ absconded.

\section{Discussion}

Heart failure (HF) is a major public health problem worldwide. Available data suggests that the morbidity due to HF is significant in many parts of the world. However, the etiologies vary across the globe. This cross-sectional study revealed that progressive hypertensive heart disease is the leading cause of heart failure in our centre while hypertensive diabetics have higher risk of developing heart failure than subjects with hypertension alone. This is similar to the study by 


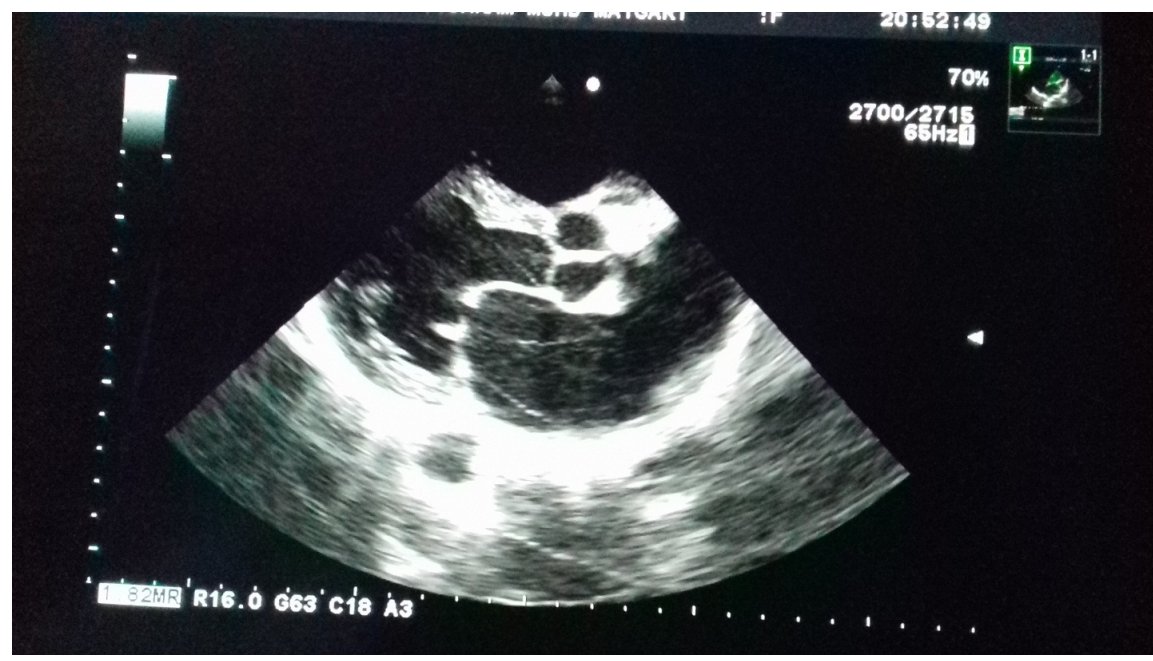

Figure 3. Two dimensional echocardiography showing rheumatic heart disease with thickened mitral valve and dilated left atrium.

Damasceno et al. on the causes, treatment, and outcome of acute heart failure in 1006 Africans from nine countries [7]. Cardiomyopathies are the second most predominant aetiology for heart failure after hypertension in our centre contributing 33.6\%. The two most important cardiomyopathies in this study were the peripartum cardiomyopathy $(22.6 \%)$ and the idiopathic dilated cardiomyopathy (11\%) a finding similar to what was previously reported [7]. Peripartum cardiomyopathy occurs between the start of the last month of pregnancy and the end of the fifth month of delivery in the absence of pre-existing signs, symptoms or history of heart failure and has evidence of left ventricular systolic dysfunction on echocardiography [14]. Peripartum cardiomyopathy is more frequent in sub-Saharan Africa than in developed countries [15].

Rheumatic heart disease remains a major cause of heart failure in Africa. In this study, it is the third cause of heart failure in our centre accounting for 7.3\% though it was lower than what was previously reported in the THESUS-HF study [7]. Ischemic heart disease has always been considered a rarity, however, studies have shown an increasing incidence. Mahmoud et al. in Kano reported 46 cases of ischaemic heart disease over a period of 5 years [16]. Our study, however, recorded only 5 cases of myocardial infarction over a period of one year. This is probably due to the rural nature of our centre where most patients are from poor socioeconomic background with increased physical activities compared to Kano a commercial city. In addition, Aminu Kano teaching hospital receives bulk of the patient referrals from many centres across the northwest region of Nigeria. Only one patient had a diagnosis of Cor-pulmonale constituting $0.3 \%$. This is similar to what was reported by Eyo et al. in their study on Echocardiographic pattern of acquired heart diseases in Nigeria [17].

Several precipitating factors have been identified that may contribute to HF hospitalizations, viz: arrhythmia, myocardial ischemia, pneumonia, hypertension, worsening renal failure, and lifestyle modification or medication 
non-compliance [18] [19]. In this study the most common precipitating factor for heart failure was chest infection which is followed by non-adherence to prescription. Urinary tract infection (UTI) was reported as the third ranked precipitating factor for heart failure. Combinations of UTI with chest infection (4.8\%) as well as UTI with arrhythmia $(2.3 \%)$ and chest infection with arrhythmia $(7.6 \%)$ were recorded in this study. Infections generally precipitate heart failure through increase in metabolic demand from fever. In addition patients with heart failure are susceptible to respiratory tract infection because of diminished ability of congested lungs to expel respiratory secretions [20]. Prescription non-adherence was previously reported as one of the important precipitants of heart failure as observed in this study [17] [18]. Other precipitants in this study were arrhythmia and anaemia with uncontrolled hypertension. Arrhythmia was particularly common among patients with rheumatic heart disease while anaemia with uncontrolled hypertension were common among patients with impaired renal function. Alejandro et al. in their study on precipitating factors leading to decompensation of chronic heart failure in the elderly patient reported that arrhythmia was the precipitant in one quarter of their patients [21]. Ischaemic heart disease as precipitant of heart failure in this study was recorded in $0.8 \%$, while endocarditis was reported in only in $0.6 \%$. It is important to identify these precipitating factors and address them to reduce hospitalization rate, the duration of hospital stay and morbidity as well as to improve outcome of heart failure.

Majority of the patients were admitted in NYHA class IV, with extensive pedal oedema and reduced left ventricular systolic function implying a delayed presentation. However, 63.3\% were discharged home in NYHA class II with significant improvement in left ventricular ejection fraction particularly those admitted in heart failure with reduced ejection fraction. This is perhaps due to strict prescription adherence while on admission. Nevertheless, $31.4 \%$ were discharged in NYHA class IV with ejection fraction of less than $40 \%$, especially among the elderly admitted with reduced ejection fraction. There was no significant change in the diastolic functions among the study population on admission and at discharge. This could be due to the short duration of hospital stay which was averagely about 7 days. Impaired relaxation pattern of left ventricular diastolic dysfunction was the predominant pattern of diastolic dysfunction among patients with progressive hypertensive heart disease as well as among hypertensive-diabetic patients, probably driven by left ventricular hypertrophy [22]. Restrictive left ventricular diastolic dysfunction was the commonest form of diastolic dysfunction among patients with peripartum cardiomyopathy and idiopathic dilated cardiomyopathy. This finding could be due to elevated left ventricular end diastolic pressure as previously reported by Bruno et al. [23]. However, among patients with priormyocardial infarction, impaired relaxation was the only form of diastolic dysfunction observed. Though this category of patients was very few in this study, the pattern of diastolic dysfunction exhibited could be due to reduced myocardial compliance associated with ischaemia [24]. Majority of patients with 
progressive hypertensive heart disease in failure had preserved left ventricular ejection fraction. This may be due to hypertension induced left ventricular hypertrophy as previously reported by Aurigemma et al. [25]. In this study, patients with peripartum and idiopathic dilated cardiomyopathy had predominantly reduced left ventricular systolic function. This is due to left ventricular dilatation and reduced myocardial contractility associated with peripartum and dilated cardiomyopathy. About $90 \%$ of the patients were discharged home with improved clinical condition while $5.4 \%$ died on admission suggesting that prompt proper evaluation and treatment will result in satisfactory short-term outcome.

\section{Conclusion}

The study revealed that progressive hypertensive heart disease is the leading cause of heart failure, followed by peripartum cardiomyopathy while idiopathic dilated cardiomyopathy and rheumatic heart disease were ranked third and fourth cause of heart failure respectively. Though myocardial infarction is on the increase, it was found to be the fifth cause of heart failure. The commonest precipitating factor for heart failure in this study was chest infection followed by non-adherence to prescription while urinary tract infection was the third. Substantial proportion of heart failure hospitalizations was associated with preventable precipitating factors. Therefore, knowledge of potential precipitating factors may help to optimize treatment. The short-term outcome of heart failure patients admitted in our centre was generally good. This study is however limited by failure to assess long term outcome.

\section{Limitations of the Study}

1) Follow-up study was not done to assess the long term outcome in our heart failure patients.

2) Adequate assessment of left ventricular diastolic function was limited by non-inclusion of pulmonary venous flow and both lateral and medial tissue Doppler parameters.

3) BNP assay among the study population was not done.

\section{References}

[1] Ponikowski, P., Voors, A.A., Anker, S.D., et al. (2016) ESC Guidelines for the Diagnosis and Treatment of Acute and Chronic Heart Failure. The Task Force for the Diagnosis and Treatment of Acute and Chronic Heart Failure of the European Society of Cardiology (ESC) Developed with the Special Contribution of the Heart Failure Association (HFA) of the ESC. European Journal of Heart Failure, 18, 891-975. https://doi.org/10.1002/ejhf.592

[2] Hunt, S.A., Abraham, W.T., Chin, M.H., et al. and the American college of Cardiology Foundation; American Heart Association. (2009) Focused Update Incorporated into the ACC/AHA 2005 Guidelines for the Diagnosis and Management of Heart Failure in Adults: A Report of the American College of Cardiology Foundation/American Heart Association Task Force on Practice Guidelines Developed in 
Collaboration with the International Society for Heart and Lung Transplantation. Journal of the American College of Cardiology, 53, e1-e9. https://doi.org/10.1016/j.jacc.2008.11.013

[3] Yancy, C.W., Jessup, M., Bozkurt, B., et al. (2013) 2013 ACCF/AHA Guideline for the Management of Heart Failure: Executive Summary. Circulation, 128, 1810-1852.

[4] Ponikowski, P., Voors, A.A., Anker, S.D., et al. (2016) Authors/Task Force Members. 2016 ESC Guidelines for the Diagnosis and Treatment of Acute and Chronic Heart Failure: The Task Force for the Diagnosis and Treatment of Acute and Chronic Heart Failure of the European Society of Cardiology (ESC) Developed with the Special Contribution of the Heart Failure Association (HFA) of the ESC. European Heart Journal, 37, 2129-2200. https://doi.org/10.1093/eurheartj/ehw128

[5] Damasceno, A., Cotter, G., Dzudie, A., Sliwa, K. and Mayosi, B.M. (2007) Heart Failure in Sub-Saharan Africa: Time for Action. Journal of the American College of Cardiology, 50, 1688-1693. https://doi.org/10.1016/j.jacc.2007.07.030

[6] Mayosi, B.M. (2007) Contemporary Trends in the Epidemiology and Management of Cardiomyopathy and Pericarditis in Sub-Saharan Africa. Heart, 93, 1176-1183. https://doi.org/10.1136/hrt.2007.127746

[7] Damasceno, A., Mayosi, B.M., Sani, M., et al. (2012) The Causes, Treatment, and Outcome of Acute Heart Failure in 1006 Africans from 9 Countries. Archives of Internal Medicine, 172, 1386-1394. https://doi.org/10.1001/archinternmed.2012.3310

[8] Dokainish, H., Teo, K., Zhu, J., et al. (2017) Global Mortality Variations in Patients with Heart Failure: Results from the International Congestive Heart Failure (INTER-CHF) Prospective Cohort Study. The Lancet Global Health, 5, e665-e6672. https://doi.org/10.1016/S2214-109X(17)30196-1

[9] Lucas, C., Johnson, W., Hamilton, M.A., et al. (2000) Freedom from Congestion Predicts Good Survival despite Previous Class IV Symptoms of Heart Failure. American Heart Journal, 140, 840-847. https://doi.org/10.1067/mhj.2000.110933

[10] MacIntyre, K., Capewell, S., Stewart, S., et al. (2000) Evidence of Improving Prognosis in Heart Failure Trends in Case Fatality in 66,547 Patients Hospitalized Between 1986 and 1995. Circulation, 102, 1126-1131.

https://doi.org/10.1161/01.CIR.102.10.1126

[11] Levy, D., Kenchaiah, S., Larson, M.G., et al. (2002) Long Term Trends in the Incidence of and Survival with Heart Failure. The New England Journal of Medicine, 347, 1397-1402. https://doi.org/10.1056/NEJMoa020265

[12] Henes, J. and Rosenberger, P. (2016) Systolic Heart Failure: Diagnosis and Therapy. Current Opinion in Anesthesiology, 29, 55-60. https://doi.org/10.1097/ACO.0000000000000270

[13] Mckee, P.A., Castelli, W.P., Mcnamara, P.M. and Kannel, W.B. (1971) The Natural History of Congestive Heart Failure: The Framingham Study. The New England Journal of Medicine, 285, 1441-1446.

[14] Hilfiker-Kleiner, D. and Sliwa, K. (2014) Pathophysiology and Epidemiology of Peripartum Cardiomyopathy. Nature Reviews Cardiology, 11, 364-370. https://doi.org/10.1038/nrcardio.2014.37

[15] Fett, J.D., Christie, L.G., Carraway, R.D. and Murphy, J.G. (2005) Five-Year Prospective Study of the Incidence and Prognosis of Peripartum Cardiomyopathy at a Single Institution. Mayo Clinic Proceedings, 80, 1602-1606. https://doi.org/10.4065/80.12.1602

[16] Sani, M.U., Adamu, B., Mijinyawa, M.S., et al. (2006) Ischaemic Heart Disease in 
Aminu Kano Teaching Hospital, Kano Nigeria: A 5 Year Review. Nigerian Journal of Medicine, 15, 128-131. https://doi.org/10.4314/njm.v15i2.37095

[17] Ekpe, E.E., Ikpe, M.C. and Umoh, I. (2015) Echocardiographic Pattern of Acquired Heart Diseases in Nigeria. Nigerian Medical Journal, 56, 253-257. https://doi.org/10.4103/0300-1652.169699

[18] Chin, M.H. and Goldman, L. (1997) Factors Contributing to the Hospitalization of Patients with Congestive Heart Failure. American Journal of Public Health, 87, 643-648. https://doi.org/10.2105/AJPH.87.4.643

[19] Ghali, J.K., Kadakia, S., Cooper, R. and Ferlinz, J. (1988) Precipitating Factors Leading to Decompensation of Heart Failure: Traits among Urban Blacks. Archives of Internal Medicine, 148, 2013-2016. https://doi.org/10.1001/archinte.1988.00380090087021

[20] Formiga, F., Chivite, D., Manito, N., et al. (2007) Hospitalization Due to Acute Heart Failure. Role of the Precipitating Factors. International Journal of Cardiology, 120, 237-241. https://doi.org/10.1016/j.ijcard.2006.10.004

[21] Diaz, A., Ciocchini, C., Esperatti, M., Becerra, A., Mainardi, S. and Farah, A. (2011) Precipitating Factors Leading to Decompensation of Chronic Heart Failure in the Elderly Patient in South-American Community Hospital. Journal of Geriatric Cardiology, 8, 12-14. https://doi.org/10.3724/SP.J.1263.2011.00012

[22] Adamu, G.U., Katibi, A.I., George, O., Omotoso, A.B.O. and Araoye, A.M. (2010) Prevalence of Left Ventricular Diastolic Dysfunction in Newly Diagnosed Nigerians with Systemic Hypertension: A Pulsed Wave Doppler Echocardiographic Study. African Health Sciences, 10, 177-182.

[23] Pinamonti, B., Dilena, A., Sinagra, G., Camerini, F. and the Heart Muscle Disease Study Group (1993) Restrictive Ventricular Filling Pattern in Dilated Cardiomyopathy Assessed by Doppler Echocardiography: Clinical, Echocardiographic and Haemodynamics Correlations and Prognostic Implications. Journal of the American College of Cardiology, 22, 808-815.

[24] El Din Behairy, N.H., Homos, M., Ramadan, A. and El Sayed Gouda, S.O. (2014) Evaluation of Left Ventricle Diastolic Dysfunction in Ischemic Heart Disease by CMR: Correlation with Echocardiography and Myocardial Scarring. The Egyptian Journal of Radiology and Nuclear Medicine, 45, 1099-1104. https://doi.org/10.1016/j.ejrnm.2014.07.007

[25] Aurigemma, G.P., Zile, M.R. and Gaasch, W.H. (2006) Contractile Behavior of the Left Ventricle in Diastolic Heart Failure: With Emphasis on Regional Systolic Function. Circulation, 113, 296-304. https://doi.org/10.1161/CIRCULATIONAHA.104.481465 